\title{
The M3Y Double Folding Dissipative Model in Agreement with Precise Fusion Cross Sections
}

\author{
I. I. Gontchar, M. V. Chushnyakova \\ Physics and Chemistry Department, Omsk State Transport University, Omsk, Russia \\ Email: vigichar@hotmail.com
}

Received 2013

\begin{abstract}
Large numbers of precision fusion excitation functions were fitted in the literature using the nucleus-nucleus interaction potential having the Woods-Saxon shape. The diffuseness of this potential fusion ranges from 0.75 to $1.5 \mathrm{fm}$. This is much larger than the value of $0.65 \mathrm{fm}$ required by the elastic scattering data. Trying to resolve this contradiction we develop the dissipative trajectory model based on the density-dependent M3Y NN-forces folded with the nuclear matter distribution. Resulting potential possesses the normal diffuseness about $0.65 \mathrm{fm}$. With this potential we reach the agreement with the data for ${ }^{16} \mathrm{O}+{ }^{208} \mathrm{~Pb},{ }^{28} \mathrm{Si}+{ }^{208} \mathrm{~Pb},{ }^{32} \mathrm{~S}+{ }^{208} \mathrm{~Pb}$ reactions within $5 \%$.
\end{abstract}

Keywords: Heavy Ion Fusion; M3Y NN-Forces; Double Folding Potential; Fusion Excitation Function

\section{Introduction}

Nucleus-nucleus collision is the main process from which we obtain our knowledge about the properties of the nuclei [1]. The fusion process, during which two complex nuclei are converted into one excited compound-nucleus, is the most probable result of such collision as the energy of the relative motion of the nuclei exceeds the Coulomb barrier [2]. During last two decades many interesting features of the fusion process between two complex nuclei were discovered [3,4]. Measured capture (fusion) cross sections are conventionally analyzed in the framework of the coupled-channels model [3, 5]. The nucleus-nucleus interaction potential is the crucial element of this model. Usually, the Woods-Saxon (WS) ansatz is used (see, e.g., Equation (2) in [5] or Equation (1) in [6]). Its most important ingredient is the diffuseness which finally defines the properties of the fusion barrier: the larger is the diffuseness the lower is the barrier and the larger is its radius (i.e. the distance between the centers of two spherical nuclei at which the barrier is located).

Systematic analysis of the experimental capture excitation functions in [4] showed that the values of the diffuseness ranging between 0.75 and $1.5 \mathrm{fm}$ are needed in order to reproduce the data. This is much larger than the value of $0.65 \mathrm{fm}$ which is required by the elastic scattering data [7]. It was discussed in [4] that these abnormally large diffusenesses might be due to some unaccounted dynamical effects.

Developing this suggestion, we try to analyze the pre- cision experimental capture excitation functions using the dissipative trajectory model with the surface friction $[8,9]$. Most features of the model are specified in detail in [6]. Therefore, in the present contribution we give only qualitative description and concentrate on the comparison to the data and the conclusions which can be drown from this comparison.

\section{The Model}

Within the framework of our model, the fictitious Brownian particle with the reduced mass move experiencing the action of the conservative, dissipative, and stochastic forces. We consider the collision process at the energies well above the Coulomb barrier. Therefore the quantum effects like tunneling and channels couplings are of no importance and are not accounted for.

The collision of two spherical nuclei is considered. That is why we account only for two degrees of freedom corresponding to the radial and orbital motion. Our previous study [6] showed that the radial motion is rather fast. Therefore we apply the corresponding equations in the non-Markovian shape considering the retarding friction and colored noise for the radial momentum. These equations finally are reduced to the system of 5 stochastic differential equations with the white noise for an auxiliary variable. The diffusion coefficient is related to the friction coefficient and to the temperature by the Einstein relation (see, e.g., Equation (49) in [10]). The nuclear potential energy is calculated using our code [11] based on the double-folding of the M3Y density dependent 
$\mathrm{NN}$-forces with the nuclear densities [12]. Initial conditions for the numerical modeling have been carefully chosen to guarantee they do not influence the calculated cross sections.

In our model the nucleus-nucleus potential defines both the conservative and dissipative forces between colliding nuclei. Therefore, we present it in Figure 1 for the case of ${ }^{16} \mathrm{O}+{ }^{208} \mathrm{~Pb}$ reaction. The M3Y double folding potential (solid line) is compared here with the WoodsSaxon profile possessing abnormally large diffuseness [4] and the standard Gross-Kalinowski potential [8]. We see that our potential differs significantly from the predecessors.

In the M3Y double folding potential the nuclear matter density is a crucial ingredient. This density was taken to be proportional to the nuclear charge density. For the latter distribution the Woods-Saxon profile (see Equation (34) in [11]) was used. Two parameters of the profile are related via the root mean square radius. The latter was taken from [13]. The diffuseness for all the projectile nuclei was taken as $0.5 \mathrm{fm}$. The diffuseness of the target nucleus ${ }^{208} \mathrm{~Pb}$ was taken as $0.6 \mathrm{fm}$.

\section{Results}

We calculated the fusion (capture) cross sections for the following three reactions for which the high precision data are available: ${ }^{16} \mathrm{O}+{ }^{208} \mathrm{~Pb}$ [14], ${ }^{28} \mathrm{Si}+{ }^{208} \mathrm{~Pb}$ [15], ${ }^{32} \mathrm{~S}+$ ${ }^{208} \mathrm{~Pb}$ [16]. In Figure 2 we present the experimental fusion excitation functions (upper panel) and the resulting correlation between the data and the calculated cross sections (lower panel) versus the relative collision energy. The latter is equal to the ratio of the collision energy to the typical Coulomb barrier height $\mathrm{B}_{\mathrm{Z}}=\mathrm{Z}_{\mathrm{P}} \mathrm{Z}_{\mathrm{T}} /\left(\mathrm{A}_{\mathrm{P}}^{1 / 3}+\right.$ $\left.A_{T}^{1 / 3}\right)$. The cross sections for this figure are computed using the single barrier penetration model (see Equations $(22,24)$ in [6]) which does not include any dynamics. The M3Y double folding potential was used. Here we clearly see the essence of the abnormally large diffuseness problem: the calculation with the potential whose typical diffuseness is $0.65 \mathrm{fm}$ strongly overestimates the data. On the other hand, the Woods-Saxon profile, which fits the fusion data, possesses the large diffuseness which is in disagreement with the one required by the elastic scattering data [7].

In Figure 3 we present the calculated fusion excitation functions (upper panel) and the resulting correlation between the data and the calculated cross sections. Here modeling was performed including the retarding friction and corresponding colored noise.

The friction coefficient strength factors were $2.0 \times 10^{-2}$ $\mathrm{zs} / \mathrm{MeV}$ for the radial motion and $1.0 \times 10^{-4} \mathrm{zs} / \mathrm{MeV}$ for the orbital motion. The correlation time for the noise was chosen to be an ajustable parameter. Results presented in Figure $\mathbf{3}$ are obtained with the following values of this time: 0.1 zs for ${ }^{16} \mathrm{O}+{ }^{208} \mathrm{~Pb}, 0.15$ zs for ${ }^{28} \mathrm{Si}+{ }^{208} \mathrm{~Pb}$ and ${ }^{32} \mathrm{~S}+{ }^{208} \mathrm{~Pb}$. Overall agreement of the calculations to the data in Figure 3(b) is very good: among almost 50 points only in 4 cases the ratio deviate from unity by more than $5 \%$. Note that at low values of the relative collision energy our approach is expected to underestimate the cross sections because the quantum and coupling effects are ignored. This is the reason why the ratio of the cross sections systematically decreases for ${ }^{16} \mathrm{O}+{ }^{208} \mathrm{~Pb}$ reaction as the relative collision energy goes to unity.

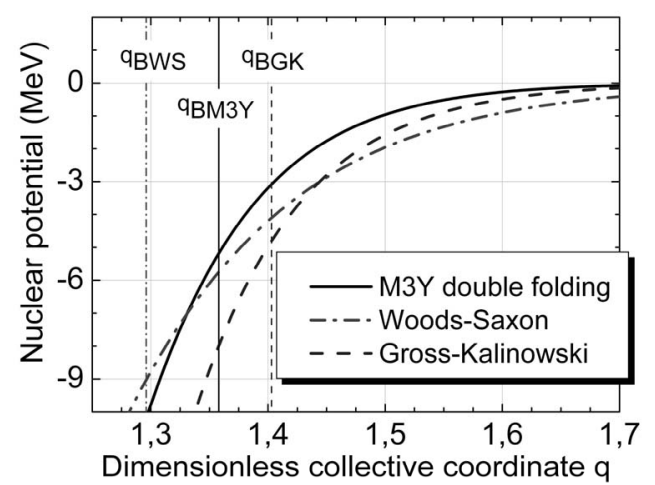

Figure 1. Different nuclear potentials versus dimensionless center-of-mass distance for ${ }^{16} \mathrm{O}+{ }^{208} \mathrm{~Pb}$ reaction; the vertical lines indicate the barrier radii.
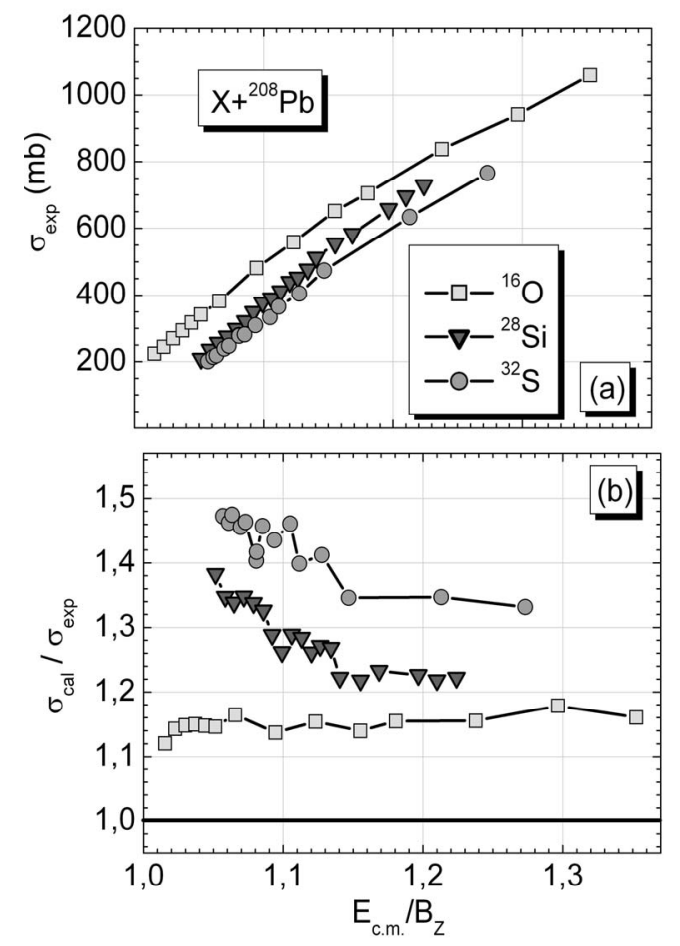

Figure 2. (a) The experimental excitation functions (the error bars are inside the symbols); (b) The ratio of the capture cross section calculated using the single barrier penetration model to the experimental cross section versus the relative collision energy. 


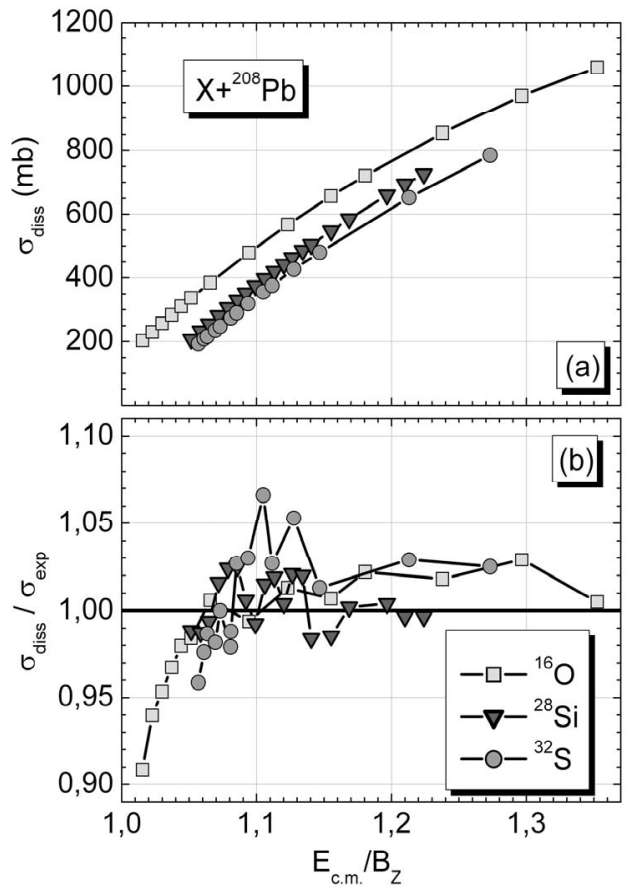

Figure 3. (a) The excitation functions calculated using the M3Y double folding dissipative model; (b) The ratio of the capture cross section calculated using the M3Y double folding dissipative model to the experimental cross section versus the relative collision energy.

\section{Conclusions}

The Woods-Saxon shape for the nucleus-nucleus interaction potential was used extensively in the literature in order to analyze the precision fusion excitation functions [4,14-16]. Excellent fit was achieved with the diffuseness of this potential ranging from 0.75 up to $1.5 \mathrm{fm}$. This is significantly larger than the value of $0.65 \mathrm{fm}$ with which the elastic scattering cross sections are usually successfully fitted [7].

This contradiction stimulated us to compose the dissipative trajectory model based on the density-dependent M3Y NN-forces folded with the nuclear matter distribution. Resulting potential possesses the normal diffuseness about $0.65 \mathrm{fm}$.

Within the framework of our approach the nucleusnucleus collision process is modeled as the motion of the fictitious Brownian particle experiencing the action of the conservative, dissipative, and stochastic forces. Since only the collision energies well above the Coulomb barrier are considered, the quantum effects like tunneling and channels couplings are not accounted for. Possible retarding character of the dissipation and non-Markovian nature of the noise are included.

Using this model we reached the agreement with the data for ${ }^{16} \mathrm{O}+{ }^{208} \mathrm{~Pb},{ }^{28} \mathrm{Si}+{ }^{208} \mathrm{~Pb},{ }^{32} \mathrm{~S}+{ }^{208} \mathrm{~Pb}$ reactions within $5 \%$. This is done with the universal strength coefficient of radial friction $2.0 \times 10^{-2} \mathrm{zS} / \mathrm{MeV}$ and with the correlation time of the noise equal to $0.1 \mathrm{zs}$ for ${ }^{16} \mathrm{O}+{ }^{208} \mathrm{~Pb}$ reaction and $0.15 \mathrm{zs}$ for ${ }^{28} \mathrm{Si}+{ }^{208} \mathrm{~Pb}$ and ${ }^{32} \mathrm{~S}+{ }^{208} \mathrm{~Pb}$ reactions.

This advance together with successful description of the capture cross sections for ${ }^{16} \mathrm{O}+{ }^{92} \mathrm{Zr}$ and ${ }^{16} \mathrm{O}+{ }^{144} \mathrm{Sm}$ reactions obtained in [6] seems giving a hope to resolve the long standing problem of apparently large diffuseness of the nucleus-nucleus potential.

\section{REFERENCES}

[1] P. Fröbrich and R. Lipperheide, "Theory of Nuclear Reactions," Oxford Studies in Nuclear Physics, Vol. 18, Oxford University Press, 1996.

[2] A. C. Berriman, D. J. Hinde, M. Dasgupta, C. R. Morton, R. D. Butt and J. O. Newton, "Unexpected Inhibition of Fusion in Nucleus-Nucleus Collision,” Nature, Vol. 413, 2001, pp. 144-147. doi:10.1038/35093069

[3] M. Dasgupta, D. J. Hinde, N. Rowley and A. M. Stefanini, "Measuring Barriers to Fusion," Annual Review of Nuclear and Particle Science, Vol. 48, 1998, pp. 401-461. doi:10.1146/annurev.nucl.48.1.401

[4] O. Newton, R. D. Butt, M. Dasgupta, D. J. Hinde, I. I. Gontchar, C. R. Morton and K. Hagino, "Systematic Failure of the Woods-Saxon Nuclear Potential to Describe Both Fusion and Elastic Scattering: Possible Need for a New Dynamical Approach to Fusion,” Physical Review C, Vol. 70, 2004, 024605.

[5] K. Hagino, N. Rowley and A. T. Kruppa, “A Program for Coupled-Channels Calculations with All Order Couplings for Heavy-Ion Fusion Reactions," Computer Physics Communications, Vol. 123, No. 1-3, 1999, pp. 143-152. doi:10.1016/S0010-4655(99)00243-X

[6] M. V. Chushnyakova and I. I. Gontchar, "Heavy Ion Fusion: Possible Dynamical Solution of the Problem of the Abnormally Large Diffuseness of the Nucleus-Nucleus Potential,” Physical Review C, in press.

[7] R. A. Broglia and A. Winther, "Heavy Ion Reaction Lecture Notes, Volume I: Elastic and Inelastic Reactions," Benjamin/Cummings Publishing Company, San Francisco, 1981.

[8] D. H. E. Gross and H. Kalinowski, "Friction Model of Heavy-Ion Collision,” Physics Reports, Vol. 45, 1978, pp. 175-210. doi:10.1016/0370-1573(78)90031-5

[9] P. Fröbrich, "Fusion and Capture of Heavy Ions above the Barrier: Analysis of Experimental Data with the Surface Friction Model,” Physics Reports, Vol. 116, 1984, pp. 337- 400. doi:10.1016/0370-1573(84)90162-5

[10] P. Fröbrich and I. I. Gontchar, "Langevin Description of Fusion Deep-Inelastic Collisions and Heavy-Ion Induced Fission,” Physics Reports, Vol. 292, 1998, pp. 131-238. doi:10.1016/S0370-1573(97)00042-2

[11] I. I. Gontchar and M. V. Chushnyakova, "A C-Code for the Double-Folding Interaction Potential of Two Spherical Nuclei," Computer Physics Communications, Vol. 181, 2010, pp. 168-182. doi:10.1016/j.cpc.2009.09.007 
[12] Dao T. Khoa, G. R. Satchler and W. von Oertzen, "Nuclear Incompressibility and Desnity Dependent NN Interactions in the Folding Model for Nucleus-Nucleus Potentials,” Physical Review C, Vol. 56, 1997, pp. 954-969. doi:10.1103/PhysRevC.56.954

[13] I. Angeli, “A Consistent Set of Nuclear rms Charge Radii: Properties of the Radius Surface R(N,Z) ," Atomic Data and Nuclear Data Tables, Vol. 87, 2004, pp. 185-206. doi:10.1016/j.adt.2004.04.002

[14] C. R. Morton, A. C. Berriman, M. Dasgupta, D. J. Hinde, J. O. Newton, K. Hagino and I. J. Thompson, "Coupled-Channels Analysis of the ${ }^{16} \mathrm{O}+{ }^{208} \mathrm{~Pb}$ Fusion Barrier
Distribution,” Physical Review C, Vol. 60, No. 044608, 1999.

[15] D. J. Hinde, C. R. Morton, M. Dasgupta, J. R. Leigh, J. C. Mein and H. Timmers, "Competition Between Fusion-Fis- sion and Quasi-Fission in the Reaction ${ }^{28} \mathrm{Si}+{ }^{208} \mathrm{~Pb}$," Nuclear Physics, Vol. A592, 1995, pp. 271-289

[16] D. J. Hinde, M. Dasgupta, N. Herrald, R. G. Nielson, J. O. Newton and M.A. Lane, "Isotopic Dependence of Fusion Barrier Energies in Reactions Forming Heavy Elements,” Physical Review C, Vol. 75, No. 054603, 2007. 